\title{
Effect of Ginkgo biloba Extract Ingestion on Plasma Total Cortisol Levels during an Oral Glucose Tolerance Test in Normal Glucose Tolerant Individuals
}

\author{
George B. Kudolo \\ Department of Clinical Laboratory Sciences, University of Texas Health Science Center, San Antonio, USA \\ Email: Kudolo@uthscsa.edu
}

Received 27 June 2014; revised 30 July 2014; accepted 11 August 2014

Copyright (C) 2014 by author and Scientific Research Publishing Inc.

This work is licensed under the Creative Commons Attribution International License (CC BY). http://creativecommons.org/licenses/by/4.0/

(c) (i) Open Access

\begin{abstract}
Protracted periods of increased cortisol production, as may be seen in an acute illness, may lead to transient hyperglycemia. Increasing evidence suggests that cortisol may then mediate the development of insulin resistance and potentially lead to the development of overt diabetes. Evidence in animal studies also suggests that under conditions of stress Ginkgo biloba extract could reduce plasma cortisol production and so the primary aim of this study was to determine the effect of Ginkgo biloba extract ingestion on plasma cortisol production during an acute period of glucose challenge. Healthy non-diabetic, glucose tolerant volunteers $(n=30,(10 / 20, M / F)$; age, $45.7 \pm 9.9$ years old) completed a randomized, double-blind, placebo-controlled crossover study when they ingested Ginkgo biloba extract $(120 \mathrm{mg} /$ day as a single dose) and placebo during each 3-month arm. A standard $75 \mathrm{~g}$ oral glucose tolerance test was performed at the end of each cycle and blood was collected and used to measure plasma glucose, insulin, c-peptide and cortisol. Fasting plasma cortisol was significantly lower after the Ginkgo biloba cycle than the placebo cycle (326 \pm 149 vs. $268 \pm 121 \mathrm{nmol} / \mathrm{L}$, respectively; $p=019$ ). The plasma cortisol area under the curve during the 2-hour test $\left(\mathrm{AUC}_{0-2}\right)$ was also significantly lower after ingestion of the Ginkgo biloba cycle compared to the placebo $(668 \pm 265 \mathrm{vs}$. $530 \pm 213 \mathrm{nmol} / \mathrm{L} / \mathrm{h}$, respectively; $p<0.001)$. It is concluded that the ingestion of Ginkgo biloba extract has effect on the hypothalamic-pituitary-adrenal axis leading to reduced basal cortisol levels and reduced cortisol production in response to acute hyperglycemic challenge.
\end{abstract}

\section{Keywords}

Ginkgo biloba Extract, Cortisol, Acute Hyperglycemia 


\section{Introduction}

The hypothalamic-pituitary (HPA) axis is characterized by a circadian rhythm with a maximum cortisol production in early morning $(8-10 \mathrm{am})$ and the lowest levels found in the evening [1]. It is generally believed that during periods of high metabolic demands, such as in the morning after an overnight fast, high cortisol levels ensue which leads to increased blood glucose via gluconeogenesis and glycogenolysis [2]. It is known that sustained hypercortisolemia, produced under a variety of stressful conditions, such as trauma and depression may promote insulin resistance and the associated complications [2]-[6]. Antidepressants and anxiolytic drugs may be used for the treatment and management of stress and they do show significant reduction in cortisol production and stress reactivity [2] [7]. However, the attendant adverse reactions including fatalities, particularly with the tricyclic antidepressants [8] [9], have led people to explore the use of alternative therapies, including the use of nutraceuticals like Ginkgo biloba extract [10].

For over a decade, we have studied the effect of Ginkgo biloba extract on several parameters including pancreatic $\beta$-cell function and whole body insulin sensitivity in normal glucose tolerant individuals, those with impaired glucose tolerance and people suffering from type 2 diabetes mellitus (T2DM) [11]-[13]. During these studies the volunteers completed monthly questionnaires and were encouraged to provide other general comments about how they felt after ingesting the Ginkgo biloba extract. The participants commonly reported increased wellbeing and being "less stressed up". This current report is the result of measuring total cortisol in the same stored plasma $\left(-86^{\circ} \mathrm{C}\right)$ in which plasma insulin and C-peptide were previously measured in normal, healthy non-diabetic volunteers. The volunteers participated in a double-blind placebo control crossover study in which they ingested $120 \mathrm{mg}$ of Ginkgo biloba extract as a single dose or a placebo daily for three months. The oral glucose tolerance test (OGTT) with 75 g glucose was administered at the end of each treatment arm to 1) confirm the absence of diabetes and 2) examine quantitative pancreatic $\beta$-cell function. Because there are already studies showing that Ginkgo biloba extract regulates adrenal cortical hormone production in cell culture [14] and in intact stress-induced rats [15] the driving hypothesis was that the feeling of wellbeing reported by volunteers after ingesting Ginkgo biloba extract was due to reduced cortisol production. It was further hypothesized that the ingestion of Ginkgo biloba extract would significantly protect the HPA axis even after an acute glucose challenge.

\section{Study Subjects and Methods}

\subsection{Study Subject and Protocol}

Volunteers for the study were enrolled in response to advertisements on bulletin boards around the University campus and endocrine clinics of the South Texas Veterans Health Care System, Audie L. Murphy Division. The experimental protocols were approved by the Institutional Review Board of the University of Texas Health Science Center, the Scientific Advisory Board of the Frederic C. Bartter General Clinical Research Center (GCRC), and the Research and Development Committee of the South Texas Veterans Health Care System, Audie L. Murphy Division. Written, informed and voluntary consent was obtained from all participants. All the volunteers were screened, using clinical laboratory blood tests, including complete blood counts and a comprehensive metabolic panel, before enrollment into the study. Body weights for all individuals enrolled had been stable for at least 3 months prior to beginning the study. Potential participants without major medical problems (major cardiovascular, hepatic and other endocrine diseases) as determined by routine medical history, physical examination, blood and urine tests were included in the study. A pregnancy test was performed in all women of reproductive potential, and pregnant or nursing women were excluded. Volunteers with anemia $[\mathrm{Hb}<12 \mathrm{~g} / \mathrm{dl}$ (female) or $<13 \mathrm{~g} / \mathrm{dl}$ (male)] were also excluded. The subjects were advised to abstain from diets and other dietary supplements rich in fish oils (such as EPA Plus ${ }^{\mathrm{TM}}$ and Omega $3^{\mathrm{TM}}$ ), and other antioxidants such as quercetin, and vitamins A, C and E. The participants were instructed not to take any platelet inhibiting drugs, such as nonsteroidal anti-inflammatory drugs (NSAID), for at least 10 days preceding the end of each arm of the study as platelet function tests were performed in another part of the study protocol.

\subsection{Study Design}

All participants (30) were asked to come to the GCRC, after an overnight fast (10 - 12 hours), where they were weighed, their heights taken (for determination of body mass index), body composition profile performed using 
a bioelectrical impedance method (Spectrum Lightweight II, RJL Systems Instruments), electrocardiography performed, and blood pressure checked and they were randomly assigned to receive either a placebo or Ginkgo biloba extract (120 mg as a single dose). The Ginkgo biloba extract was a 50:1 standardized extract (Whole Health Inc.) with a potency of approximately $27 \%$ flavone glycosides (a combination of isorhamnetin, myricetin, kaempferol and quercetin) and 6\% terpenes (ginkgolides, A, B, C and bilobalides), as analyzed by high performance liquid chromatography (HPLC). Next, the subjects filled out a 2-page questionnaire documenting their current state of health, symptoms and medications and their general health. The research staff verified the information provided and all the volunteers underwent the standard $75 \mathrm{~g}$ glucose 2-hour OGTT, at the start of the experiment to verify the absence or presence of diabetes mellitus using clinical recommendations of the American Diabetes Association [16], and also to monitor their pancreatic $\beta$-cell function. Blood samples were collected during the basal state for the measurement of the comprehensive metabolic panel, including the total lipid, complete blood count and coagulation profiles and the measurement of cortisol, glucose, insulin and c-peptide. The subjects returned to the GCRC monthly for follow-up visits when they donated blood for clinical laboratory tests, completed questionnaires to document any changes in their health, symptoms, and any significant changes in their diet. The subjects also returned unused study capsules, which were counted to estimate the compliance rate. No detailed subject food records were required for this study. At the end of each $3^{\text {rd }}$ month, the OGTT was repeated. Thus, each subject undertook the OGTT three times but only the results of the second and third tests were used for analysis (the first was used to confirm the absence of diabetes and admittance into the study).

\subsection{Analytical Techniques}

All the clinical laboratory tests were performed in the Chemistry Laboratory of the South Texas Veterans Health Care System, Audie L. Murphy Division. Glycosylated hemoglobin $\left(\mathrm{HbA}_{1 \mathrm{c}}\right)$ was determined using high-pressure liquid chromatography technique using a Daimet Glycosylated Hemoglobin Analyzer (Bio-Rad Laboratories, Hercules, CA). Plasma glucose concentration during the OGTT was determined using the glucose oxidase method on a Beckman Glucose Analyzer II (Beckman, Fullerton, CA). Plasma insulin, C-peptide and cortisol levels were determined using radioimmunoassay kits (Diagnostic System Laboratories, Inc. Webster, TX, USA).

\subsection{Statistical Analysis}

A mathematical calculation of the required sample size was based upon the mean and standard deviation (SD) of the basal cortisol levels in the two arms of the study group (Ginkgo biloba extract and placebo cycles). A minimum sample size of 27 was required to detect significant difference with a type 1 error $(\alpha=0.05)$, a power (1- $\beta)$ of 0.8 and at 0.5 effect size. The area under the glucose and cortisol curves during the 2-hour OGTT (AUC ${ }_{0-2}$ ) was calculated using the trapezoid rule. The Student's paired $t$-test was used to compare values during the placebo and Ginkgo biloba cycles, using the Add-in Statistical package of the Excel software (Office for Mac 2004). $P$ values $<0.05$ were considered significant.

\section{Results}

The study participants had normal glucose tolerance according to the clinical recommendation of American Diabetes Association [16] and Table 1 provides a summary of other selected diagnostic test results (from the comprehensive metabolic panel) on the last day of each 3-month treatment arm during the 6-month study. Thirty study participants completed the study and were very compliant in taking the supplements and placebos. The ingestion of $120 \mathrm{mg}$ (as a single dose) had no significant effect on any of the laboratory test panels performed, including the lipid panel and the coagulation tests.

Ingestion of Ginkgo biloba extract caused a significant reduction in the fasting plasma cortisol levels as well as the cortisol production during the 2-hour OGTT $\left(\mathrm{AUC}_{0-2}\right)$.

The individual subject cortisol response $\left(\mathrm{AUC}_{0-2}\right)$ is illustrated in Figure 1. The participants did not report any adverse events during the course of the studies.

\section{Discussion}

The results of this study indeed showed that ingestion of Ginkgo biloba extract does affect the HPA axis, a confirmation of what has been demonstrated in isolated adrenocortical cells [14] and in intact rats [15]. Ingestion of 
Table 1. Clinical characteristics of study participants $(n=30 ; 10 / 20(M / F))$ after ingesting a placobo and Ginkgo biloba extract during each 3-month cycle.

\begin{tabular}{|c|c|c|c|}
\hline \multirow{2}{*}{ Parameter (Units) (Ref. range/cutoff) } & \multicolumn{3}{|c|}{ Treatment } \\
\hline & Placebo & Ginkgo biloba Extract & $P$ value \\
\hline Compliance rate (\%) & $93 \pm 0.1$ & $94.0 \pm 0.1$ & 0.286 \\
\hline Age (years) & $45.7 \pm 9.9$ & & \\
\hline Body Mass Index $\left(\mathrm{kg} / \mathrm{m}^{2}\right)$ & $29.8 \pm 6.2$ & $29.9 \pm 6.2$ & 0.350 \\
\hline Systolic blood pressure $(\mathrm{mm} \mathrm{Hg})(<120)$ & $128 \pm 15$ & $123 \pm 12$ & $0.046^{*}$ \\
\hline Diastolic blood pressure (mm Hg) $(<80)$ & $76 \pm 11$ & $74 \pm 9$ & 0.083 \\
\hline Hemoglobin $\mathrm{A}_{1 \mathrm{c}}(\%)(4.2$ - 5.8) & $5.3 \pm 0.4$ & $5.5 \pm 0.4$ & 0.190 \\
\hline Fasting Insulin (mU/L) (2 - 25) & $8 \pm 5$ & $10 \pm 7$ & $0.025^{*}$ \\
\hline Fasting C-peptide $(\mu \mathrm{g} / \mathrm{L})(<4)$ & $2.0 \pm 1.6$ & $2.5 \pm 1.3$ & $0.049^{*}$ \\
\hline Total cholesterol $(\mathrm{mg} / \mathrm{dl})(<5.18)$ & $4.84 \pm 1.01$ & $5.02 \pm 0.98$ & 0.121 \\
\hline Triglycerides $(\mathrm{nmol} / \mathrm{L})(<2.03)$ & $1.27 \pm 0.82$ & $1.37 \pm 0.76$ & 0.232 \\
\hline HDL-Cholesterol (nmol/L) (>0.91) & $1.30 \pm 0.26$ & $1.32 \pm 0.28$ & 0.298 \\
\hline LDL-Cholesterol (mg/dl) $(<3.37)$ & $3.00 \pm 0.75$ & $3.08 \pm 0.80$ & 0.288 \\
\hline Fibrinogen (g/L) (1.98 - 5.24) & $2.89 \pm 0.71$ & $2.94 \pm 0.63$ & 0.318 \\
\hline PT (s) $(10.8-13.4)$ & $11.9 \pm 1.3$ & $11.9 \pm 1.2$ & 0.419 \\
\hline PTT(s) (20.0 - 31.6) & $28.5 \pm 2.4$ & $28.9 \pm 1.1$ & 0.380 \\
\hline Fasting glucose (nmol/L) (3.89 - 5.83) & $5.05 \pm 0.50$ & $5.22 \pm 0.50$ & 0.067 \\
\hline Glucose $\mathrm{AUC}_{0-2}(\mathrm{nmol} / \mathrm{L} / \mathrm{h})$ & $14.93 \pm 2.61$ & $15.15 \pm 2.55$ & 0.277 \\
\hline Fasting cortisol (nmol/L) (138 - 635) & $326 \pm 149$ & $268 \pm 121$ & $0.019^{*}$ \\
\hline Cortisol $\mathrm{AUC}_{0-2}(\mathrm{nmol} / \mathrm{L} / \mathrm{h})$ & $668 \pm 265$ & $530 \pm 213$ & $0.001^{*}$ \\
\hline
\end{tabular}

All values $=$ mean \pm SD and values between placebo and Ginkgo biloba cycles were compared using paired t-tests. $\mathrm{P}$ values $<0.05$ were considered significant $\left({ }^{*}\right)$.

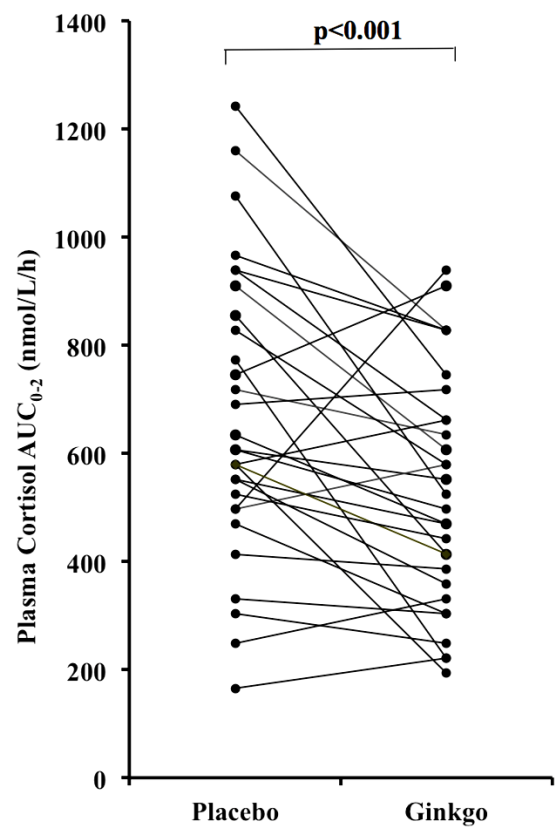

Figure 1. Individual responses after a 75 g-glucose challenge after ingesting a placebo and Ginkgo biloba extract. 
Gingko biloba extract not only caused a significant reduction in the fasting (or basal) plasma cortisol levels but it also reduced cortisol production during the 2-hour glucose challenge $\left(\mathrm{AUC}_{0-2}\right)$, a condition simulating acute hyperglycemia, compared to ingestion of the placebo. These results have major implications for the way that stress-related pathologies may be addressed nutritionally. As a glucocorticoid, cortisol is released in response to a variety of stress stimuli including critical illnesses leading to hyperglycemia. In fact, stress hyperglycemia [17] is a common occurrence in patients with acute and critical illnesses, even in the absence of diabetes mellitus, which was originally thought to be beneficial. However, it is now believed that, at any given severity, they face worse consequences than those with pre-existing diabetes [17].

Presumably protracted periods of stress and chronic cortisol production could conceivably produce hyperglycemia, increasing accumulation of body fat and enhancing insulin resistance [6]. This chronic stress condition may also lead not only to depression [4] but also to the development of impaired glucose tolerance and eventually overt T2DM and the associated complications such as dyslipidemia, stroke and hypertension [18]. Any intervention that would reduce chronic hypercortisolemia must therefore be considered.

The results of the present studies indicate that the ingestion of Ginkgo biloba extract might serve to reduce cortisol production when challenged with a high glucose load and this adds to our continuing efforts to characterize the whole body effect of ingesting Ginkgo biloba extract. We have already reported that the ingestion of $120 \mathrm{mg}$ Ginkgo biloba extract daily as a single dose reduced platelet in vitro aggregation mediated by collagen, decreased plasma thromboxane $\mathrm{B}_{2}$ synthesis in vitro and accompanied by decreased urinary 11-dehydrothromboxane $B_{2}$ excretion in both normal glucose tolerant and type II diabetic subjects [19]. We have also reported the reduction of malondialdehyde levels (a marker for oxidative stress) in washed platelets, harvested from healthy, non-diabetic and T2DM subjects, even when augmented with arachidonic acid [20]-[22]. Furthermore, we have reported an increase in pancreatic $\beta$-cell production of insulin and C-peptide especially in T2DM subjects with pancreatic exhaustion [12]. In this case it is possible for Ginkgo biloba extract to reverse the deleterious stressinduced cortisol effect on the pancreatic $\beta$-cell [23]. Taken together, there are several positive aspects of ingesting Ginkgo biloba extract.

Ginkgo biloba extract contains approximately 27\% flavonol glycosides (mainly isorhamnetin, myricetin, kaempferol and quercetin) and 6\% terpenes (ginkgolides, A, B, C and bilobalides). The flavonol glycosides have been said to be responsible for the free radical scavenging character of the extract. Quercetin (3,3',4',5,7-pentahydroxyflavone), one of the major constituents of the flavonol glycosides, has been independently shown to lower cortisol levels in rodent models of stress [24] [25] and is found in many foods items such as onions, apples, berries, tea, grapes, red wine and prickly pear. In fact, the prickly pear (Opuntia Streptacantha), which is used in Mexican folklore to treat T2DM, has been shown to have anti-hyperglycemic effects in rats [26]. The ginkgolides are known to bind to the platelet activating factor receptor in several biological systems, with ginkgolide B having the strongest affinity [27]. The addition of ginkgolide B to adrenocortical cells isolated from rodents have already been shown to cause a reduction in the production of cortisol in tissue culture [14] which may lead to the conclusion that perhaps it is this fraction that may interact directly with the HPA axis to cause a decrease in cortisol production. So both fractions of the Ginkgo biloba extract would appear to contribute to the reduction of cortisol reduction.

The major limitation of this study was that specialized stress tests were not performed and this could the subject of future studies. Other future studies could examine the effect of Ginkgo biloba extract on cortisol production in people with chronic hyperglycemia and T2DM. More importantly further research is warranted to determine the mechanism by which the two major components, the flavonol glycosides and the ginkgolides, interact with target points along the HPA axis to alter cortisol production.

\section{Conclusion}

It is possible that functional foods containing the active ingredients found in the Ginkgo biloba extract may be beneficial to several groups including people with marked insulin resistance, such as the obese [28] who also tend to have hypercortisolemia. Diabetes has become an epidemic worldwide in all age groups, with the prevalence rising faster in the youth and this may be related to diet and behavior [29]. In Latino youth for example, metabolic syndrome has been found to be associated with higher morning serum cortisol [30]. It is clear then that proper diet, dietary supplements or functional foods rich in the active ingredients found in Ginkgo biloba extract may be advantageous in reducing the chances of developing diabetes. It is also probably wise to consider 
foods that are rich in these active ingredients as part of food donations to people after traumatic events and natural disasters when blood cortisol is known to be elevated [31].

\section{Acknowledgements}

The technical assistance of Ryan Elrod and Alexis Haase is gratefully acknowledged. The nursing and dietetic care provided by the staff of the Frederic C. Bartter General Clinical Research Center at the South Texas Veterans Health Care Systems-Audie Murphy Division is appreciated. This study was supported by funds from NIH grants M01-RR-01346 and R01-AT-00832 (National Center for Complementary and Alternative Medicine, NCCAM). Its contents are solely the responsibility of the authors and do not necessarily represent the official views of the NCCAM, or the National Institutes of Health.

\section{References}

[1] Akerstedt, T. and Levi, L. (1978) Circadian Rhythms in the Secretion of Cortisol, Adrenaline and Noradrenaline. European Journal of Clinical Investigation, 8, 57-58. http://dx.doi.org/10.1111/j.1365-2362.1978.tb00811.x

[2] Hucklebridge, F.H., Clow, A., Abeyguneratne, T., Huezo-Diaz, P. and Evans, P. (1999) The Awakening Cortisol Response and Blood Glucose Levels. Life Sciences, 64, 931-937. http://dx.doi.org/10.1016/S0024-3205(99)00019-3

[3] Bhagwagar, Z., Hafizi, S. and Cowen, P.J. (2005) Increased Salivary Cortisol after Waking in Depression. Psychopharmacology, 182, 54-57. http://dx.doi.org/10.1007/s00213-005-0062-z

[4] Stokes, P.E. (1995) The Potential Role of Excessive Cortisol Induced by HPA Hyperfunction in the Pathogenesis of Depression. European Neuropsychopharmacology, 5, 77-82. http://dx.doi.org/10.1016/0924-977X(95)00039-R.

[5] Reynolds, R.M., Javier, L., Strachan, W.W.J., Braun, A., Fowkes, F.G.R., Lee, A.J., Frier, B.M., Seckl, J.R., Walker, B.R. and Price, J.F. on behalf of the Edinburgh Type 2 Diabetes Study (ET2DS) Investigators (2010) Elevated Fasting Plasma Cortisol Is Associated with Ischemic Heart Disease and Its Risk Factors in People with Type 2 Diabetes: The Edinburgh Type 2 Diabetes Study. Journal of Clinical Endocrinology \& Metabolism, 95, 1602-1608. http://dx.doi.org/10.1210/jc.2009-2112

[6] Heraclides, A., Chandola, T., Witte, D.R. and Brunner, E.J. (2009) Psychosocial Stress at Work Doubles the Risk of Type 2 Diabetes in Middle-Aged Women Evidence from the Whitehall II Study. Diabetes Care, 32, 2230-2205. http://dx.doi.org/10.2337/dc09-0132

[7] Vermetten, E., Vythiliingan, M., Schmahl, C., Kloet, C., Southwick, S.M., Charney, D.S. and Bremner, J.D. (2006) Alterations in Stress Reactivity after Long-Term Treatment with Paroxetine in Women with Posttraumatic Stress Disorder. Annals of the New York Academy of Sciences, 1071, 184-202. http://dx.doi.org/10.1196/annals.1364.014

[8] Jimmink, A., Caminada, K., Hunfeld, N.G. and Touw, D.J. (2008) Clinical Toxicology of Citalopram after Acute Intoxication with Sole Drug or in Combination with Other Drugs: Overview of 26 Cases. Therapeutic Drug Monitoring, 30, 365-371. http://dx.doi.org/10.1097/FJC.0b013e3181379ef6

[9] Amitai, Y. and Frischer, H. (2006) Excess Fatality from Desipramine in Children and Adolescents. Journal of the American Academy of Child Adolescent Psychiatry, 45, 54-60. http://dx.doi.org/10.1097/01.chi.0000184931.26176.4a

[10] Itil, T. and Martoarano, D. (1995) Natural Substances in Psychiatry (Ginkgo biloba in Dementia). Psychopharmacology Bulletin, 31, 147-158.

[11] Kudolo, G.B. (2000) The Effect of 3-Month Ingestion of Ginkgo biloba Extract on Pancreatic $\beta$-Cell Function in Response to Glucose Loading in Healthy Volunteers. Journal of Clinical Pharmacology, 40, 647-654. http://dx.doi.org/10.1002/j.1552-4604.2000.tb05991.x

[12] Kudolo, G.B. (2001) The Effect of 3-Month Ingestion of Ginkgo biloba Extract (EGb 761) on Pancreatic $\beta$-Cell Function in Response to Glucose Loading in Individuals with Non-Insulin Dependent Diabetes Mellitus. Journal of Clinical Pharmacology, 41, 600-611. http://dx.doi.org/10.1177/00912700122010483

[13] Kudolo, G.B., Wang, W., Elrod, R., Barrientos, J., Haase, A. and Blodgett, J. (2006) Short-Term Ingestion of Ginkgo Biloba Extract (EGb 761) Does Not Alter Whole Body Insulin Resistance in the Non-Diabetic, Pre-Diabetic or Type 2 Diabetic Subjects-A Short Randomized Double-Blind Placebo-Controlled Crossover Study. Clinical Nutrition, 25, 123-134. http://dx.doi.org/10.1016/j.clnu.2005.10.001

[14] Amri, H., Drieu, K. and Papadopoulus, V. (1997) Ex Vivo Regulation of Adrenal Cortical Cell Steroid and Protein Synthesis, in Response to Adrenocorticotropic Hormone Stimulation, by Ginkgo biloba Extract (EGb761) and Isolated Ginkgolide B. Endocrinology, 138, 5415-5426.

[15] Walesiuk, A., Trofimiuuk, E. and Brasko, J.J. (2006) Ginkgo biloba Normalizes Stress- and Corticosterone-Induced Impairment of Recall in Rats. Pharmacological Research, 53, 123-128. http://dx.doi.org/10.1016/j.phrs.2005.09.007 
[16] American Diabetes Association: Clinical Practice Recommendation (2005) Standards of Medical Care in Diabetes. Diabetes Care, 28, S4-S36. http://dx.doi.org/10.2337/diacare.28.suppl_1.S4

[17] Dugan, K.M., Braithwaite, S.S. and Preiser, J.C. (2009) Stress Hyperglycemia. Lancet, 373, 1798-1807. http://dx.doi.org/10.1016/S0140-6736(09)60553-5

[18] Rosmond, R. (2005) Role of Stress in the Pathogenesis of the Metabolic Syndrome. Psychoneuroendocrinology, 30, 1-10. http://dx.doi.org/10.1016/j.psyneuen.2004.05.007

[19] Kudolo, G.B., Dorsey, S. and Blodgett, J. (2003) Effect of the Ingestion of Ginkgo biloba Extract on Platelet Aggregation and Urinary Prostanoid Excretion in Healthy and Type 2 Diabetic Subjects. Thrombosis Research, 108, 151-160. http://dx.doi.org/10.1016/S0049-3848(02)00394-8

[20] Kudolo, G.B., Wang, W., Barrientos, J., Elrod, R. and Blodgett, J. (2004) The Ingestion of Ginkgo biloba Extract Inhibits Arachidonic Acid-Mediated Platelet Aggregation and Thromboxane $\mathrm{B}_{2}$ Production in Healthy Subjects. Journal of Herbal Pharmacotherapy, 4, 13-26. http://dx.doi.org/10.1080/J157v04n04_02

[21] Kudolo, G.B., Wang, W., Dorsey, S. and Blodgett, J. (2003) Oral Ingestion of Ginkgo biloba Extract Reduces Thiobarbituric Acid Reacting (TBAR) Substances in Washed Platelets of Healthy Subjects. Journal of Herbal Pharmacotherapy, 4, 1-15. http://dx.doi.org/10.1080/J157v03n04_01

[22] Kudolo, G.B., Delaney, D. and Blodgett, J. (2005) Short-Term Oral Ingestion of Ginkgo biloba Extract (EGb 761) Reduces Malondialdehyde Levels in Washed Platelets of Type 2 Diabetic Subjects. Diabetes Research and Clinical Practice, 68, 29-38. http://dx.doi.org/10.1016/j.diabres.2004.08.007

[23] Byung-Wan, L., Jun, H., Yim, H.J., Park, J.B., Woo, H.J. and Yoo, H.J. (2010) Dysfunctional Pancreatic $\beta$-Cells of Critical Stress Play a More Prominent Role in the Development of Stress Diabetes in Critically Burned Korean Subjects. Metabolism-Clinical and Experimental, 59, 1307-1315. http://dx.doi.org/10.1016/j.metabol.2009.11.022

[24] Kawabata, K., Kawai, Y. and Terao, J. (2010) Suppressive Effect of Quercetin on Acute Stress-Induced HypothalamicPituitary-Axis Response in Wistar Rats. Journal of Nutritional Biochemistry, 21, 374-380. http://dx.doi.org/10.1016/j.jnutbio.2009.01.008

[25] Bhutada, P., Mundhada, Y., Bansod, K., Ubgade, A., Quazi, M., Umathe, S. and Mundhada, M. (2010) Reversal by Quercetin of Corticotrophin Releasing Factor Induced Anxiety- and Depression-Like Effect in Mice. Progess in NeuroPsychopharmacology \& Biological Psychiatry, 34, 955-960. http://dx.doi.org/10.1016/j.pnpbp.2010.04.025

[26] Adolfo, A.C. and Helmut, W. (2011) Anti-Hyperglycemic Effect of Opuntia streptacantha Lem. Journal of Ethnopharmacology, 133, 940-943. http://dx.doi.org/10.1016/j.jep.2010.11.022

[27] Kudolo, G.B. and Harper, M.J. (1989) Characterization of Platelet-Activating Factor Binding Sites on Uterine Membranes from Pregnant Rabbits. Biology of Reproduction, 41, 587-603. http://dx.doi.org/10.1095/biolreprod41.4.587

[28] Roberts, C., Troop, N., Connan, F., Treasure, J. and Campbell, I.C. (2007) The Effects of Stress on Body Weight: Biological and Psychological Predictors of Change in BMI. Obesity, 15, 3045-3055. http://dx.doi.org/10.1038/oby.2007.363

[29] Wild, S., Roglic, G., Green, A., Sicree, R. and King, H. (2004) Global Prevalence of Diabetes: Estimates for the Year 2000 and Projections for 2030. Diabetes Care, 27, 1047-1053. http://dx.doi.org/10.2337/diacare.27.5.1047

[30] Weigensberg, M.J., Toledo-Corral, C.M. and Goran, M.I. (2008) Association between the Metabolic Syndrome and Serum Cortisol in Overweight Latino Youth. Journal of Clinical Endocrinology \& Metabolism, 93, 1372-1378. http://dx.doi.org/10.1210/jc.2007-2309

[31] Song, Y.Q., Zhou, D.F. and Wang, X.D. (2008) Increased Serum Cortisol and Growth Hormone Levels in Earthquake Survivors with PTSD or Subclinical PTSD. Psychoneuroendocrinology, 33, 1155-1159. http://dx.doi.org/10.1016/j.psyneuen.2008.05.005 
Scientific Research Publishing (SCIRP) is one of the largest Open Access journal publishers. It is currently publishing more than 200 open access, online, peer-reviewed journals covering a wide range of academic disciplines. SCIRP serves the worldwide academic communities and contributes to the progress and application of science with its publication.

Other selected journals from SCIRP are listed as below. Submit your manuscript to us via either submit@scirp.org or Online Submission Portal.
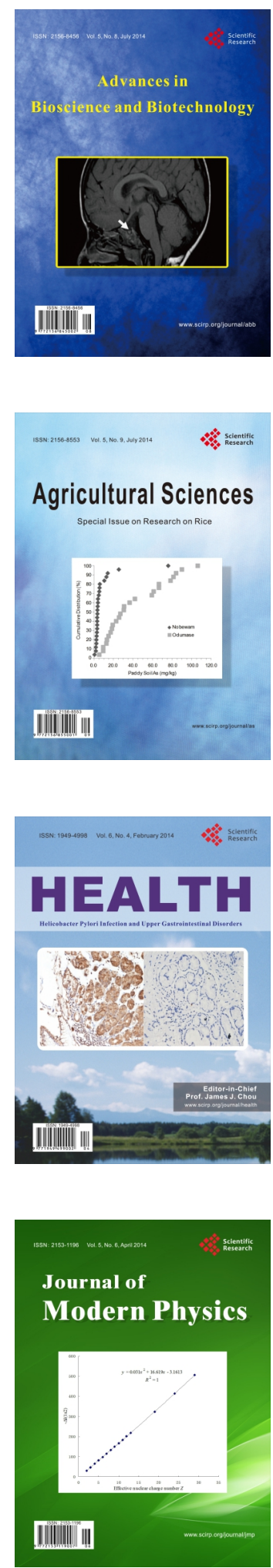
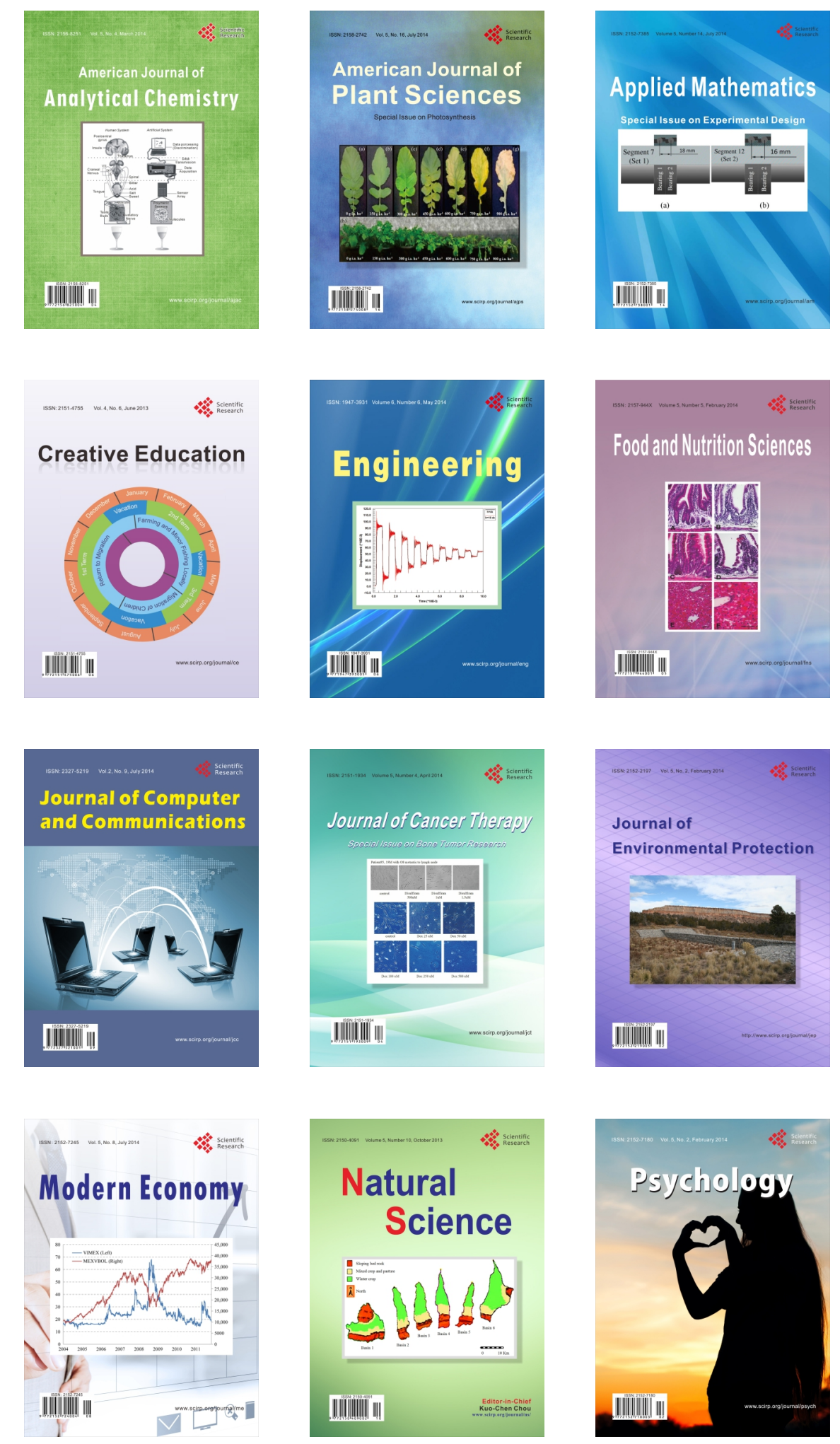